\title{
Inducción de procesos cognitivos a partir de referentes semánticos basados en el reconocimiento y priming visual con desenlace trágico*
}

[Versión en español]

Induction of Cognitive Processes from Semantic Referents Based on Recognition and Visual Priming with Tragic Outcome

Indução de processos cognitivos de referentes semânticos com base no reconhecimento e priming visual com resultado trágico

Recibido mayo 30 de 2018. Aceptado agosto 28 de 2019.

\author{
Ricardo Arrubla-Sánchez** \\ https://orcid.org/0000-0003-1548-8195
}

Colombia

Para citar este artículo:

Arrubla-Sánchez, Ricardo (2020).

Inducción de procesos cognitivos a partir de referentes semánticos basados en el reconocimiento y priming visual con desenlace trágico. Ánfora, 27(48), 43-66. DOI: https://doi.org/10.30854/anfv27.n48.2020.668 Universidad Autónoma de Manizales. ISSN 0121-6538/ e-ISSN 2248-6941

\section{Resumen}

Objetivo: establecer si la inducción de procesos cognitivos, a partir de referencias semánticas, permite una mejora en la respuesta de los conceptos de la cultura de la legalidad como instrumentos de modelos educativos para prevenir conductas disociales y criminales, en estudiantes con déficit de atención y trastornos de conducta. Metodología: estudio

* Este artículo es el resultado del proyecto de Investigación: Estudio cualitativo para el diseño de un modelo de manejo de la conducta antisocial juvenil en planteles educativos desde un curso de formación de la razón jurídica y práctica, iniciado en el año 2016 en Planteles Educativos de Secundaría con el propósito de ofrecer soluciones pedagógicas a las instituciones que tienen estudiantes con problemas de aprendizaje y trastornos de conducta disocial.

** Magíster en administración de organizaciones. Comunicador social organizacional. Docente Investigador de la Fundación Universitaria del Área Andina. Doctorante en Ciencias de la Educación Universitaria de Cuauhtémoc. Director del grupo de investigación Interdisciplinar en Estudios de Desarrollo Social y Humano, Fundación Universitaria del Área Andina. Correo: rarrubla@areandina.edu.co 
exploratorio descriptivo-explicativo, con dos grupos focales integrados por estudiantes de $11^{\circ}$ grado que se sometieron a una entrevista semiestructurada, a la aplicación de la Escala de conducta antisocial y criminal y formaron parte de los talleres del grupo de control. Resultados: se encontró que de los 19 ítems evaluados el procedimiento mostró una mejora en 8 aspectos, 4 ítems de empeoramiento y 6 ítems que no mostraron cambios. Se evidenció que la inducción de procesos a partir de referentes semánticos refleja una forma general de aprendizaje en los sistemas de representación perceptiva que puede producir modificaciones cognitivas profundas. Conclusiones: la inducción semántica a través del reconocimiento y priming visual en el aprendizaje de la cultura de la legalidad, es un método que incide en la estructuración cognitiva del estudiante y para su efectividad depende del grado de superposición perceptiva entre el primer estímulo y los estímulos subsiguientes.

Palabras-clave: Aprendizaje; Cognición; Reconocimiento; Inducción semántica; Desenlace trágico.

\begin{abstract}
Objective: to establish if the induction of cognitive processes - from semantic references - allows an improvement in the response of Culture of Legality concepts as instruments of educational models to prevent disruptive and criminal behaviors in students with attention deficit and conduct disorders. Methodology: a descriptiveexplicative exploratory study, with two focus groups composed of 11th-grade students who underwent a semi-structured interview, the application of the Antisocial and Criminal Conduct Scale and were part of the control group workshops. Results: from the 19 items evaluated, the procedure showed an improvement in 8 aspects, worsening in 4 items and 6 items that did not show any changes. It evidenced that the induction of processes from semantic referents reflects a general way of learning in systems of perceptive representation that can produce deep cognitive modifications. Conclusions: semantic induction through recognition and visual priming in the learning of Culture of Legality, is a method that affects the cognitive structuring of the student and its effectiveness depends on the degree of perceptual overlap between the first stimulus and subsequent stimuli.
\end{abstract}

Keywords: Learning; Cognition; Recognition; Semantic Induction; Tragic outcome. 


\section{Resumo}

Objetivo: estabelecer se a indução de processos cognitivos, com baseem referências semânticas, permite melhorar a resposta dos conceitos da cultura da legalidade como instrumentos de modelos educacionais para prevenir comportamentos disociais e criminais, em alunos com déficit de atenção e distúrbios de comportamento. Metodologia: estudo exploratório-descritivo-explicativo, com dois grupos focais compostos por alunos do $11^{\circ}$ ano submetidos a uma entrevista semiestruturada, à aplicação da Escala de Comportamento Anti-Social e Criminal e fizeram parte das oficinas do grupo controle. Resultados: constatou-se que dos 19 itens avaliados, o procedimento apresentou melhora em 8 aspectos, 4 itens de piora e 6 itens que não apresentaram alterações. Foi evidenciado que a indução de processos a partir de referentes semânticos reflete uma forma geral de aprendizagem nos sistemas de representação perceptiva que podem produzir profundas modificações cognitivas. Conclusões: a indução semântica por meio do reconhecimento e do estímulo visual na aprendizagem da cultura da legalidade, é um método que afeta a estrutura cognitiva do aluno e, para sua efetividade, depende do grau de sobreposição perceptiva entre o primeiro estímulo e os estímulos. subsequentes.

Palavras-chave: Aprendizagem; Cognição; Reconhecimento; Indução semântica; Resultado trágico. 


\section{Introducción}

Este artículo presenta una investigación de tipo exploratorio con estudiantes de grado $11^{\circ}$, que consiste en proponer un modelo de educación social especializado con base en la creación de un neuro-currículo que permite contrarrestar los efectos del trastorno de conducta disocial, los déficits de atención, el síndrome de atribución hostil y la desconexión moral.

Los jóvenes que padecen este tipo de síndromes presentan con cierta frecuencia conductas oposicionistas, desafiantes, agresivas y de desobediencia. Los alumnos con déficit y trastornos de atención fueron seleccionados para la investigación, ya que tienen mayor nivel de probabilidad de presentar dificultades al momento de su aprendizaje frente a la comprensión y aceptación de las normas y la cultura de la legalidad.

La investigación establece, en una primera etapa, que existe una ruptura entre los centros de producción de las normas y los centros de recepción y que ese desconocimiento, rechazo y dificultad conceptual, genera un impacto en su comprensión, su interés y los vuelve vulnerables a un contexto violento. También los afecta para que puedan estructurar su racionalidad jurídica y llegar a ejercer de manera plena sus derechos y la ciudadanía.

Por tal razón, se quiso indagar a partir de un segundo momento, pensando en el papel del educador y en la posibilidad de generar una solución al problema encontrado en su relación con los déficits y trastornos de atención, puesto que es una de las causas que incide para que tantos jóvenes sean procesados por delitos cada año, realidad que cuestiona los modelos educativos e institucionales para crear los referentes cognitivos apropiados que permitan inhibir la conducta dolosa o criminal en los jóvenes.

La preparación de los instrumentos y métodos de la segunda etapa se realizó con la aplicación de un pre y un post test, que permitieran relacionar la activación de los sistemas cognitivos y neurolingüísticos por medio de referentes semánticos. Para su estudio, se emplean los supuestos de la teoría de los modelos de configuración cognitiva, la relación que comporten sujeto/objeto y la estructura común de reconocimiento de la forma, el fondo y la figura del objeto, además del grado en que esas relaciones espaciales se desvían del objeto prototipo o "medio".

Los modelos de reconocimiento por la configuración cognitiva, ayudan a explicar cómo se crean los referentes mentales a nivel de significado y significante a partir de una categoría semántica. Han sido especialmente útiles en el campo del reconocimiento facial Diamond y Carey (1977) y Rhodes, Brake y Carey (1987), ya que la investigación sustenta la tesis de que el efecto más fuerte 
sobre la conducta de los jóvenes proviene del contexto cultural; así que, siguiendo la lógica de los modelos de configuración, se puede explicar cómo el grado de relación entre las normas, la ilegalidad y su incomprensión, inciden en el grado en que esas relaciones se desvían del prototipo establecido por la cultura de la legalidad, incididos por las imágenes que reciben del contexto.

Frente al tema de la atención, se siguen las teorías del psicólogo británico Broadbent (1958) quien defendía el postulado que la selección se efectúa en una fase inicial del procesamiento; y para comprender su funcionamiento, propone un modelo del sistema de atención que contiene un canal de capacidad limitada a través del cual sólo podía pasar una cierta cantidad de información. Su investigación permite determinar que los inputs sensitivos realizan un proceso de cribado para permitir que sólo pase la información que consideran más importante.

Este factor explica por qué los jóvenes son vulnerables, ya que no tienen referentes que les permitan procesar de manera racional la información que llega del contexto y sobre todo en las zonas de exclusión donde la evidencia del trastorno disocial es más crítica, por recibir mayor influencia de las subculturas y contraculturas. En el aula, su problema se convierte en una barrera de aprendizaje que se manifiesta en la aparición de una conducta de forma paralela, lo que se puede considerar con el desarrollo normo-típico.

Autores como Bloomquist y Schnell (2005), afirman que los casos de niños con conducta problemática que no se intervienen educativamente, presentan una progresión en el desarrollo conflictivo de su interacción social. Además, atribuyen dificultades en el desarrollo psicológico, debido a la carencia de competencias y habilidades en ciertas áreas. Para Greene y Oliva (2009) la carencia de habilidades cognitivas, intervienen en la comprensión del entorno social, la resolución de problemas y la capacidad para afrontar la adversidad. A su vez, se toman como evidencia los aportes hechos por Turecki y Tonner (2003), quienes proponen que las personas pueden quedar atrapadas en ciertos esquemas de pensamiento y que tales esquemas se vuelven un círculo de conflictos. Finalmente, Geddes (2010) sostiene que el apego es fundamental durante estos años para la creación de vínculos afectivos, al incidir en su equilibrio emocional y en la adaptación social.

\section{Fundamentos teóricos y conceptuales: las alteraciones cognitivas y el trastorno disocial en adolescentes}

La investigación parte de la premisa teórica sustentada por Ruchkin et al. (2002), de que los entornos violentos junto con los déficits de desarrollo cognitivo en la escuela y la disrupción familiar, inciden en la generación del trastorno 
disocial de la conducta (TDC). Por tratarse de una alteración de la personalidad que impide el cumplimiento de las normas básicas para la convivencia social y genera patrones de conducta delincuenciales, debe ser tenida en cuenta, ya que su aparición y dinámica sirve para explicar y comprender su relación con el aprendizaje aceptación/rechazo de la legalidad.

La línea argumental de teóricos como Déry et al. (1999) hacen un énfasis en identificar dificultades en las habilidades comunicativas, sociales, ejecutivas y de planeación, atención, razonamiento abstracto, juicio, auto-monitoreo y control motor. Las correlaciones clínicas más importantes establecidas a partir del trastorno disocial son el consumo de alcohol y drogas por los jóvenes, evidenciando que sus conductas tienden a estar relacionadas con la impulsividad, agresividad, búsqueda de sensaciones y narrativas violentas.

Lo anterior, incide en los siguientes aspectos: i) temperamento infantil, siendo el más característico el de adquisición de patrones oposicionistas, con bajo nivel de adaptación social; ii) factores parentales siendo el más común, una inadecuada relación paterno-filial; iii) factores familiares siendo frecuente que aparezca en hogares numerosos, con poca estabilidad o cohesión; iv) factor socioeconómico; v) los factores comunitarios, sobre todo en comunidades con elevada criminalidad, vi) y finalmente, los factores escolares, manifestándose en un pobre énfasis académico y cambios en la conducta y disciplina. Por estar asociado a factores que inciden en el aprendizaje de las reglas, las normas y la incorporación de los principios de la legalidad.

Ahora, por tratarse de un síndrome cognitivo, el factor disocial tiene hondas implicaciones en el comportamiento y en la capacidad de establecer redes semánticas y neuronales, que en los estudiantes agresivos presenta las siguientes características: i) realizan un análisis de la situación de acuerdo con sus experiencias pasadas en situaciones similares más que sobre los hechos concretos de la realidad actual; ii) tienen un mayor número de atribuciones hostiles y iii) presentan un déficit en el procesamiento de la información y en la toma de decisiones.

Al estar la memoria del individuo distorsionada por la panorámica cultural, se tienen en consideración que los problemas vienen de su parte estructural y llegan al joven que lo recepta sin barreras cognitivas ni morales claras. Este fenómeno complejo es explicado por Nadel, Campbell y Ryan (2007) demuestran que existe una activación en el hipocampo durante el desarrollo de actividades y tareas que involucran la memoria semántica y el recuerdo. Mientras que Campbell y Conway (1995) sustentan en sus trabajos la importante función que ejercen los patrones culturales en la reestructuración y las deformaciones del recuerdo. Evidenciaron estos autores que los individuos medidos en los grupos, tendían a introducir en sus contactos con las otras culturas los factores típicos de 
su folclore tribal. Del mismo modo, reorganizaron las secuencias e introducían giros en la trama que eran característicos de sus historias nativas.

Dicho fenómeno, también ocurre en las preferencias estéticas y el gusto artístico, la evolución de los estilos en música, diseño, arquitectura, dibujo y otras artes, son un testimonio de la transformación de los gustos. Una generación rechaza a la otra, o por lo menos produce una cierta mirada de sutil aprobación o un período de habituación gradual.

Para Huesmann et al. (1992), el tema se vuelve complejo cuando se trata de las creencias normativas y su aceptabilidad o no aceptabilidad de una conducta, por ser las normas los instrumentos que sirven para regular el comportamiento humano por medio de conductas admisibles o prohibidas. Desde este planteamiento, se puede afirmar en la investigación que los estudiantes que tienen mayor reacción a las normas, tienen creencias que aprueban o justifican tal comportamiento, de esta forma existe una relación directa entre los sistemas cognitivos de referencia y las conductas.

Desde esta perspectiva, los esfuerzos teóricos se han centrado en determinar las relaciones entre la conducta disocial o delictiva y la existencia de creencias y actitudes justificadoras de la misma. Según Slaby y Guerra (1988) los adolescentes infractores que tenían medida cautelar tenían una asociación significativa entre la conducta ilegal la cual se vuelve más fuerte a medida que la edad de los sujetos aumenta. Son las diferencias en las creencias normativas justificadoras de la ilegalidad las que establecen su comportamiento, que a su vez se vuelven justificadoras de la violencia y la agresión.

Al respecto, Calvete (2008) determinó en una investigación con adolescentes que el esquema de justificación de la violencia aparece como un elemento que precede a la conducta agresiva y delincuencial. Mientras que Calvete et al. (2014) orientan su esfuerzo en estudiar la relación entre el esquema de justificación de la violencia y la exposición a la violencia, llegando a la conclusión de que ser testigo de violencia es un factor predictivo de conducta agresiva.

Lo anterior, es asociado con los procesos de aprendizaje a la forma como se percibe la realidad y se actúan en ella. Por tal razón, se indaga en los patrones de rendimiento de tareas de memoria, ya que los consideran un factor fundamental que incide en la disociación y que están directamente relacionados con la recuperación explícita e implícita de información en la memoria de trabajo. La memoria explícita permite recordar experiencias, evocar situaciones y retener información como conceptos, argumentos y teorías (Schott et al., 2002; Schott et al., 2004, 2006); las experiencias pueden ser intencionales o no y pueden ser mediadas por la producción de recuerdo libre o motivado; en cambio, la memoria implícita surge a través de la recuperación no intencional de información, datos y recuerdos (Baddeley, 1999). 
Así, el denominado efecto del priming visual, funciona como un estímulo en la memoria con dominio específico, incidiendo en la forma como se representa la información o directamente en su significado (Schacter y Tulving, 1994). Otros autores como Macbeth y López-Alonso, (2007) sostienen que el priming puede ser un estímulo, semántico y perceptivo, cuyo origen proviene de las modalidades sensoriales, háptica, olfativa y gustativa.

La capacidad del estímulo depende de la posibilidad de codificación, llegando a ser superficial o profundo, cuando se efectúa a partir de la comprensión de significados. Teóricos como Nilsson, Law y Tulving (1988), defienden la duración del impacto del priming, el cual puede estar condicionado por la variable del tiempo, pero sostienen que una vez se anida en la memoria, puede quedar grabado toda la vida y sirve para completar la comprensión de nuevas raíces de palabras. Mientras que Froufe (1997) arguye que dicha relación depende del tiempo y de la adquisición y recuperación de estímulos con incidencia desigual en las personas, por otro tipo de factores como experiencias del pasado, aprendizaje significativo o reiteración del proceso.

En muchas ocasiones este tipo de nivel depende de las diferentes condiciones en las que se realizó la manipulación de los procesos de retención: Por ejemplo, el desarrollo de una clase depende del uso de los recursos didácticos, de dominio y explicación de los contenidos, de la posibilidad de retención y significación comprensiva de los datos y, finalmente, del nivel de empatía con el tema tratado.

Por eso, el decaimiento, o la recuperación de la información en la memoria puede ser acelerado o retardado, según los efectos profundos o superficiales del priming. Para Tulving y Schacter, (1990) el uso de los priming semánticos, puede verse afectado por el uso de operadores de codificación, de manera que, durante la aparición de los estímulos y la recuperación de la información, depende de la organización semántica de la misma, en la memoria. Indica ello que el priming semántico requiere de un procesamiento conceptual de los estímulos y es sensible a los cambios de información.

Sin embargo, Mulligan y Hornstein, (2000) sostienen que las manipulaciones de la atención durante el proceso de codificación no afectan el rendimiento de la memoria; incluso, algunos teóricos de esta línea de pensamiento muestran que la codificación puede ser exitosa, así se presenten factores de atención dividida. En este sentido, las investigaciones realizadas por Baron y Eustache (2001) en condición de codificación superficial e intermodal de imágenes y palabras, logrando un mayor nivel de codificación con el efecto del priming. Mientras que Felser y Roberts (2007) emplearon la visión y el tacto activo, llegando a la conclusión de que los estímulos estudiados facilitan la codificación en condiciones intramodales e intermodales. 


\section{Metodología}

Se identificó una muestra con dos grupos focales integrado por estudiantes de grado 11, segmentados así: 15 hombres entre los 16 y 17 años, 12 mujeres entre los 16 y 17 años. A estos participantes se les aplicó entrevista semiestructurada, junto con un cuestionario Escala de Conducta Antisocial y Delictiva. Luego, se realizó la intervención antes-después con grupo control, por medio de un cuestionario estructurado pre test y uno pos test. La segunda sesión se realizó en forma de taller donde son inducidos mediante procesos cognitivos a partir de referentes semánticos basados en el reconocimiento de conceptos claves de la teoría de la cultura de la legalidad, como Estado, poder, legitimidad y legalidad; para ello, se usó como referente persuasivo el priming visual con desenlace trágico. Después de esta intervención se volvió a pasar un cuestionario Post-test para comparar los resultados de los dos momentos.

El proceso de experimentación se realiza siguiendo el método de Velten que consiste en una lectura de un conjunto de frases autorreferidas que expresan vivencias agradables o desagradables e involucran situaciones con el tema en estudio en el aula y la personalidad y pensamiento negativo que tiene el estudiante respecto al mismo.

Así mismo, se incorporó el procedimiento autobiográfico de recordar experiencias personales altamente agradables o altamente desagradables, siendo parte de diferentes métodos de inducción, junto con el visionado de películas y la audición de composiciones musicales. La metodología empleada en los talleres se basaba en el MCS, en función de las preguntas que realizaban los alumnos o del debate durante el proceso de inducción semántica por medio del priming visual y de la narrativa trágica.

\section{Ámbito, población y periodo de estudio}

La población objeto de estudio fue conformada por jóvenes entre 16 y 17 años de edad, de ambos sexos, quienes cursaban grado $11^{\circ}$ de Bachillerato en el Colegio López Michelsen de estrato socio-económico 2 y quienes presentaban el síndrome de trastorno disocial de conducta.

Se revisó literatura complementaria agrupando los ejes reflexivos en las siguientes dimensiones: a) Núcleo familiar, fragmentación y violencia (Factor I). Este factor, está compuesto por cuatro ítems: A.1. Percepción del crecimiento, 
A.2 Experiencias dolorosas vividas, A.3 Experiencias de estados depresivos y A.4 Episodios de disfuncionalidad Familiar; b) La institución y sus obstáculos (Factor II). B.1. Infancia y relación con sus padres, B.2. Los problemas en la escuela, la exclusión y la falta de adaptación, B.3. Experiencias de trabajo infantil, y B.4. La calle, los referentes de grupo y las malas compañías; c) El barrio y los lazos vecinales (Factor II). C.1. Comunidad, violencia y pobreza, C. 2 El sentimiento de Seguridad/Inseguridad, C.3. Las experiencias de exclusión; d) La experiencia de Infracción Legal (Factor III). D.1. La calle como espacio sustituto, D.2. Consumo de drogas ilícitas, y D.3. Desafío a la autoridad policial; e) La Infracción Legal (Factor IV). E) Identidad y desviación, E.2. El delito, y E.3. Motivos para infringir la ley.

Para el proceso de análisis se siguieron los principios de la Teoría Fundamentada (Grounded Theory) propuesta por Strauss y Corbin (1998). Las variables continuas fueron expresadas como media y desviación estándar. Las variables del cuestionario fueron tratadas como variables cualitativas y fueron expresadas en frecuencias absolutas y porcentajes; para su comparación se utilizó el test del X2. Se consideró como valor de significación estadística una p<0,05.

Las pruebas de Pre-test y Post-test fueron realizadas a 27 estudiantes de ambos géneros; fue mayor la participación de hombres (15) sobre las mujeres (12). La muestra se seleccionó siguiendo los criterios de inclusión de la investigación, en la cual se evidenciaba que los participantes presentaban problemas de comportamiento y muchos de ellos, problemas intrafamiliares y en el contexto de su barrio.

\section{Resultados}

Los resultados que arrojan los dos momentos, antes y después de la intervención educativa (tabulados en las tablas 1 y 2 ) permiten diferenciar tres conceptos: i) cambios en las actitudes frente a la aceptación de las normas, ii) cambios en el conocimiento general de la cultura de la legalidad y iii) un conocimiento sobre las implicaciones del comportamiento delictivo. En relación con las actitudes, todos los porcentajes mejoraron después de la intervención educativa; tan sólo un ítem evidenció un empeoramiento.

Lo que más destaca de este bloque de ejes temáticos es el relativo a Costumbres, reglas y leyes, en el que sólo respondieron 31,9\% correctamente en el Pre-test y $37,8 \%$ en el Pos-test. Esto indica que, ante los demás aspectos, como Mantener la ley y el orden público, El Estado de Derecho, Administración de los asuntos públicos y Cultura de la legalidad, el uso de la inducción de referentes 
semánticos basados en el reconocimiento y priming visual con desenlace trágico generó un cambio significativo.

En cuanto a los conocimientos generales sobre la cultura de la legalidad, destaca que de 8 ítems planteados el 50\% mostró una mejoría significativa, 12,5 \% empeoró y 37,5\% no tuvo cambios. Destaca el incremento de respuestas acertadas en el Pos-test en el ítem La mayoría de las personas conocen las leyes y sus derechos individuales, con un $89,7 \%$ de aciertos y en el ítem La mayoría de las personas rechazan el comportamiento ilegal, con un 51,6\%, mostrando un cambio importante.

En el apartado de conocimientos sobre el delito y la ilegalidad, se puede establecer que es el área más resistente al cambio, tan sólo el ítem La mayoría de las personas apoyan las agencias del gobierno que hacen cumplir la ley presenta mejoría, los demás ítems constatan un empeoramiento significativo; tal es el caso del ítem Las leyes protegen los derechos de todo individuo al igual que el bienestar de la sociedad que evidenció empeoramiento, pues tan sólo el 62,2\% mostró aciertos en el Pos test.

El ítem que presenta un porcentaje más bajo es La mayoría de las personas rechazan el comportamiento ilegal, con un $16 \%$ en la prueba Pos test. En total, de los 19 ítems evaluados, se evidenció que el procedimiento mostró mejoría en 8 aspectos, 4 ítems de empeoramiento y 6 que no mostraron cambio.

Tabla 1. Características sociodemográficas de la población estudiada

\begin{tabular}{c|c|c|} 
Variables & $\begin{array}{c}\text { Pre-test } \\
(\mathrm{N}=896)\end{array}$ & $\begin{array}{c}\text { Post-test } \\
(\mathrm{N}=805)\end{array}$ \\
\hline \multicolumn{3}{|c|}{ Sexo } \\
\hline Hombre & 15 & 15 \\
\hline Mujer & 12 & 12 \\
\hline Media & Edad & 15,92 \\
\hline DS & 15,60 & 0,79 \\
\hline Nivel académico & 0,70 & Bachiller \\
\hline Grado 11 & Bachiller & 14 \\
\hline Bosa Alameda & 14 & 27 \\
\hline
\end{tabular}

Fuente: elaboración propia 
Tabla 2. Porcentaje de alumnos que responden correctamente cada pregunta en el pre y post-test

\begin{tabular}{|c|c|c|c|c|c|}
\hline Ítems & $\begin{array}{l}\text { No. } \\
\text { Correctas }\end{array}$ & $\begin{array}{c}\text { No. De } \\
\text { alumnos } \\
(\%)\end{array}$ & Valor $p$ & $\begin{array}{l}\text { \% cambio } \\
\text { respuestas } \\
\text { correctas }\end{array}$ & $\begin{array}{l}\text { Valoración } \\
\text { de la efec- } \\
\text { tividad }\end{array}$ \\
\hline \multicolumn{6}{|c|}{ Actitudes } \\
\hline \multirow{2}{*}{$\begin{array}{l}\text { Mantener la ley y el orden } \\
\text { público }\end{array}$} & Pre & $95,0 \%$ & \multirow{2}{*}{0.01} & \multirow{2}{*}{3.7} & \multirow{2}{*}{ Empeora } \\
\hline & Post & $91,6 \%$ & & & \\
\hline \multirow{2}{*}{ El Estado de Derecho } & Pre & $83,3 \%$ & \multirow{2}{*}{0.00} & \multirow{2}{*}{4.3} & \multirow{2}{*}{ Mejoraría } \\
\hline & Post & $87,1 \%$ & & & \\
\hline \multirow{2}{*}{$\begin{array}{c}\text { Administrar los asuntos } \\
\text { públicos }\end{array}$} & Pre & $67,7 \%$ & \multirow{2}{*}{0.00} & \multirow{2}{*}{19.7} & \multirow{2}{*}{ Mejoraría } \\
\hline & Post & $81,1 \%$ & & & \\
\hline \multirow{2}{*}{ Costumbres, Reglas y Leyes } & Pre & $31,9 \%$ & \multirow{2}{*}{0.03} & \multirow{2}{*}{18.49} & \multirow{2}{*}{ Mejoraría } \\
\hline & Post & $37,8 \%$ & & & \\
\hline \multirow{2}{*}{ La Cultura de la Legalidad } & Pre & $70,2 \%$ & \multirow{2}{*}{0.00} & \multirow{2}{*}{17.2} & \multirow{2}{*}{ Mejoraría } \\
\hline & Post & $82,3 \%$ & & & \\
\hline
\end{tabular}

Conocimiento sobre la cultura de la legalidad

\begin{tabular}{|c|c|c|c|c|c|}
\hline \multirow{2}{*}{$\begin{array}{l}\text { La mayoría de las personas } \\
\text { conocen las leyes y sus } \\
\text { derechos individuales. }\end{array}$} & Pre & $83,5 \%$ & \multirow{2}{*}{0.00} & \multirow{2}{*}{6.9} & \multirow{2}{*}{ Mejoraría } \\
\hline & Post & $89,7 \%$ & & & \\
\hline \multirow{2}{*}{$\begin{array}{l}\text { La mayoría de las personas } \\
\text { aceptan la ley y están } \\
\text { dispuestos a acatarla. }\end{array}$} & Pre & $90,0 \%$ & \multirow{2}{*}{0.14} & \multirow{2}{*}{2.7} & \multirow{2}{*}{$\begin{array}{c}\text { Sin } \\
\text { cambios }\end{array}$} \\
\hline & Post & $92,5 \%$ & & & \\
\hline \multirow{2}{*}{$\begin{array}{c}\text { Prevalece una actitud en } \\
\text { contra de la corrupción y de la } \\
\text { delincuencia. }\end{array}$} & Pre & $61,4 \%$ & \multirow[b]{2}{*}{0.00} & \multirow[b]{2}{*}{11.7} & \multirow[b]{2}{*}{ Mejoría } \\
\hline & Post & $69,6 \%$ & & & \\
\hline \multirow{2}{*}{$\begin{array}{c}\text { La mayoría de las personas } \\
\text { consideran que el sistema } \\
\text { judicial. }\end{array}$} & Pre & $94,4 \%$ & \multirow[b]{2}{*}{0.02} & \multirow[b]{2}{*}{0.7} & \multirow[b]{2}{*}{ Empeora } \\
\hline & Post & $93,7 \%$ & & & \\
\hline \multirow{2}{*}{$\begin{array}{l}\text { Todo miembro de la sociedad } \\
\text { tiene la oportunidad de } \\
\text { participar en la formulación, } \\
\text { la reforma, y la aplicación de } \\
\text { las leyes. }\end{array}$} & Pre & $98,3 \%$ & \multirow[b]{2}{*}{0.08} & \multirow[b]{2}{*}{1.4} & \multirow[b]{2}{*}{$\begin{array}{c}\text { Sin } \\
\text { cambios }\end{array}$} \\
\hline & Post & $96,9 \%$ & & & \\
\hline \multirow{2}{*}{$\begin{array}{l}\text { Las leyes se aplican a todos } \\
\text { por igual, incluyendo al } \\
\text { gobernante y al gobierno en } \\
\text { turno. }\end{array}$} & Pre & $29,7 \%$ & \multirow[b]{2}{*}{0.00} & \multirow[b]{2}{*}{11.7} & \multirow[b]{2}{*}{ Mejoría } \\
\hline & Post & $36,3 \%$ & & & \\
\hline
\end{tabular}




\begin{tabular}{c|c|c|c|c|c|} 
& $\begin{array}{c}\text { No. } \\
\text { Ítems }\end{array}$ & $\begin{array}{c}\text { No. De } \\
\text { alumnos } \\
\text { Correctas }\end{array}$ & Valor p & $\begin{array}{c}\text { \% cambio } \\
\text { respuestas } \\
\text { correctas }\end{array}$ & $\begin{array}{c}\text { Valoración } \\
\text { de la efec- } \\
\text { tividad }\end{array}$ \\
\hline $\begin{array}{c}\text { Las leyes protegen los } \\
\text { derechos de todo individuo } \\
\text { al igual que el bienestar de la } \\
\text { sociedad. }\end{array}$ & Pre & $72,7 \%$ & & & Sost \\
\cline { 2 - 3 } $\begin{array}{c}\text { son } \\
\text { La mayoría de las personas } \\
\text { rechazan el comportamiento } \\
\text { ilegal. }\end{array}$ & Pre & $44,9 \%$ & 0.12 & 2.9 & cambios \\
\cline { 2 - 3 } & Post & $51,6 \%$ & 0.00 & 20.9 & Mejoría \\
\hline
\end{tabular}

\section{Conocimiento sobre delito y la ilegalidad}

\begin{tabular}{|c|c|c|c|c|c|}
\hline \multirow{2}{*}{$\begin{array}{l}\text { Facilita la participación, } \\
\text { equidad y protección de cada } \\
\text { individuo. }\end{array}$} & Pre & $97,3 \%$ & \multirow{2}{*}{0.74} & \multirow{2}{*}{0.5} & \multirow{2}{*}{$\begin{array}{c}\text { Sin } \\
\text { cambios }\end{array}$} \\
\hline & Post & $96,8 \%$ & & & \\
\hline \multirow{2}{*}{$\begin{array}{c}\text { Todo miembro de la sociedad } \\
\text { tiene la oportunidad de } \\
\text { participar en la formulación, } \\
\text { la reforma, y la aplicación de } \\
\text { las leyes. }\end{array}$} & Pre & $67,0 \%$ & \multirow[b]{2}{*}{0.46} & \multirow[b]{2}{*}{4.5} & \multirow[b]{2}{*}{$\begin{array}{c}\text { Sin } \\
\text { cambios }\end{array}$} \\
\hline & Post & $64,1 \%$ & & & \\
\hline \multirow{2}{*}{$\begin{array}{l}\text { Las leyes protegen los } \\
\text { derechos de todo individuo } \\
\text { al igual que el bienestar de la } \\
\text { sociedad. }\end{array}$} & Pre & $71,5 \%$ & \multirow[b]{2}{*}{0.00} & \multirow[b]{2}{*}{14.9} & \multirow[b]{2}{*}{ Empeora } \\
\hline & Post & $62,2 \%$ & & & \\
\hline \multirow{2}{*}{$\begin{array}{c}\text { La mayoría de las personas } \\
\text { aceptan y están dispuestas } \\
\text { cumplir los deberes } \\
\text { tipificados por la ley. }\end{array}$} & Pre & $92,0 \%$ & \multirow[b]{2}{*}{0.09} & \multirow[b]{2}{*}{2.4} & \multirow[b]{2}{*}{$\begin{array}{c}\text { Sin } \\
\text { cambios }\end{array}$} \\
\hline & Post & $94,3 \%$ & & & \\
\hline \multirow{2}{*}{$\begin{array}{l}\text { La mayoría de las personas } \\
\text { rechazan el comportamiento } \\
\text { ilegal. }\end{array}$} & Pre & $20,6 \%$ & \multirow{2}{*}{0.00} & \multirow{2}{*}{28.15} & \multirow{2}{*}{ Empeora } \\
\hline & Post & $16,0 \%$ & & & \\
\hline \multirow{2}{*}{$\begin{array}{c}\text { La mayoría de las personas } \\
\text { apoyan las agencias del } \\
\text { gobierno que hacen cumplir } \\
\text { la ley. }\end{array}$} & Pre & $65,5 \%$ & \multirow[b]{2}{*}{0.00} & \multirow[b]{2}{*}{4.2} & \multirow[b]{2}{*}{ Mejoría } \\
\hline & Post & $68,7 \%$ & & & \\
\hline
\end{tabular}

\section{Fuente: elaboración propia}

Ahora bien, la inducción de procesos cognitivos por medio de referentes semánticos basados en el reconcomiendo y priming visual con desenlace trágico, es una técnica de estimulación cognitiva complementaria e importante en el aula de clase. Ello, sobre todo en contextos sociales de exclusión y déficit cultural y cuan- 
do se identifican referentes cognitivos erróneos o distorsionados que impiden el procesamiento y asimilación de nueva información.

Para el caso en estudio, el uso de referentes semánticos alusivos a la legalidad y el efecto negativo de su ausencia en la vida de un individuo sirvió de punto de apertura para generar un cambio en las estructuras cognitivas de los estudiantes, quienes al pensar en los daños que ocasiona la ilegalidad recurren a la noción de equilibración (Inheler, Sinclair y Bovet, 1975), ya que el sujeto en respuesta busca compensaciones frente a las perturbaciones del contexto.

En el ser humano, la equilibración progresiva de las estructuras cognitivas (equilibrio cognitivo) es un proceso fundamental para interactuar en la vida social, siendo evolutivo y que se puede modificar en los diferentes estadios del desarrollo cognitivo del individuo. No se puede hablar de regulación, cuando el efecto perturbador mantiene o provoca la repetición de la acción en el estudiante, sin lograr ninguna modificación.

La dinámica cognitiva del grupo focal estudiado permitió establecer que los procesos de inducción semántica son necesarios y reafirman el principio piagetiano de que las actividades cognitivas individuales adquieren su significación en las interacciones sociales reales y simbólicas que, a su vez, estructuran acciones y juicios, a partir de los diversos puntos de vista presentes en las actividades sociales. La inducción semántica es fundamental para la transformación en el aula, centrando su interés en la capacidad lingüístico-cognitiva del estudiante al momento de argumentar su posición en temas como la aceptación/rechazo de la legalidad y contribuyendo en la construcción de significados, así como en la formación de conceptos y categorías.

En ese sentido, en contextos de desigualdad y exclusión tienden a predominar relaciones sociales y estructuras psíquicas con menor autorregulación, lo cual conduce al predominio de conductas instintivas y prácticas violentas. Siendo este uno de los mayores desafíos de las instituciones de educación pública y privada. Al respecto, el sociólogo Elias (1990), contribuye en su estudio a explicar que en los fenómenos sociales existen propiedades estructurales que son incomprensibles si se presta atención solo a los individuos, así como tampoco se pueden inferir las regularidades del comportamiento individual partiendo únicamente de las propiedades grupales.

Por tal razón, desde la teoría de Siegler (1996), se valida la importancia y existencia de las estrategias como elemento fundamental en el progreso del rendimiento cognitivo. Implica ello que los proyectos educativos en contextos de exclusión Wald y Kurlander (2003), deben estar concebidos de tal manera que incluyan las normas escolares y las expectativas relacionadas con el comportamiento, las políticas administrativas, las estrategias de seguimiento, el mapeo de guiones cog- 
nitivos y pensamientos distorsionados, así como las actitudes y prácticas de los maestros.

Sin embargo, la anterior estrategia no puede ser la misma en todos los procesos de aprendizaje; sobre todo, en los contextos de exclusión y violencia tiene que estar relacionada con la incorporación de modelos mentales de recuperación de información y de comprensión transitiva del comportamiento humano. Esto, porque a la codificación y recuperación se le atribuye la principal causa de la pérdida de la información, debido al proceso que elabora la información en la mente y, en especial, a factores que pueden estar vinculados con aspectos psicológicos, emocionales o racionales (Manzanero, 2008).

Estas teorías sostienen la tesis de que la información se pierde desde el mismo momento de la transmisión, ya que los sistemas sensoriales tienen límites, barreras e intereses que orientan su atención por ciclos de interés, que pueden estar condicionados por el sistema cognitivo, el sistema racional-emocional o los estados de ánimo. Así, la información recibida es seleccionada e interpretada de acuerdo con los conocimientos previos, con la demanda de conocimientos de la tarea, el contexto, y con los esfuerzos adicionales que los estudiantes realizan en su tiempo libre para mejorar sus conocimientos, habilidades y saberes sobre unas áreas de conocimiento o interés. Durante el proceso de aprendizaje se logra una parte de la abstracción del significado hasta que la información se incorpora a las estructuras de conocimiento o se pierde en la memoria.

Por tal razón, las prácticas educativas en instituciones situadas en contexto de violencia escolar o intrafamiliar, deben emplear diferentes métodos, técnicas y didácticas de enseñanza en su pedagogía, para dilucidar y entender las causas que movilizan la conducta violenta o agresiva de los estudiantes y, lo más importante, darle prioridad al hecho de la pérdida de información en la memoria y la aparición de hábitos para la incorporación del lenguaje violento y agresivo. Estas nuevas pedagogías deben incluirse en el curso de las emociones de los estudiantes; su real condición cognitiva implica conocer la estructura semántica y su relación con la realidad y la dificultad o facilidad para la incorporación de nuevos conocimientos y saberes.

Bruner (1988) propone una inteligencia "paradigmática” y una inteligencia "narrativa”, lo cual sirve, en un terreno de manejo comunicativo, pero un compromiso más profundo con la realidad interna del estudiante, implica una neuropedagogía que analice la polaridad entre el lenguaje positivo y negativo e impida que sea mayor la incorporación del lenguaje violento, hostil y agresivo, frente al lenguaje normativo, ciudadano y político. Se trata de diagnosticar para enseñar e identificar problemas en la memoria enferma para incorporar técnicas de recuperación. Los mecanismos pedagógicos empleados deben conducir a situar al sujeto, 
ante las decisiones a tomar, en sus diferentes dilemas, pero en especial, observando que sean decisiones constructivas de sentido.

Autores como Luna y Migueles (2008) adelantan investigaciones que permiten comprender las implicaciones sociales y cognitivas de la incorporación de información falsa, y su mayor dificultad de aceptación hacia los discursos con altos grados de veracidad y de dificultad interpretativa. Sus aportes se han orientado en tres dimensiones: acciones y detalles, contenidos centrales o periféricos y tipicidad alta o baja.

Entonces, todo fracaso en el acceso a los recuerdos se establece como un fallo en los procesos de la memoria para recuperar información; pero es considerado como un proceso necesario, sano y equilibrado, en el cual el olvido de cierta información, facilita el ingreso y la incorporación de otra (Gómez-Ariza et al., 2005). Ello, induce a pensar en otras investigaciones, como las realizadas por García-Bajos, Migueles y Anderson (2009) sobre la teoría del repaso, en la cual, incorporar información que se tiene almacenada en la memoria, hace más probable que se recuerde en el momento de la prueba, pero también impide el recuerdo de otra. Se denomina mecanismo de control de tipo inhibitorio, su función es la de ayudar a seleccionar la información más útil en ciertos momentos y, por otro lado, suprime la accesibilidad a los capos neuronales donde se almacena los recuerdos.

Tales dificultades, deben ser pensadas desde una neuropedagogía que involucre diferentes componentes cognitivos; en este sentido, para Beck (2005) son tres los componentes principales: los esquemas nucleares, los esquemas intermedios y los pensamientos voluntarios/automáticos. Los esquemas cognitivos nucleares e intermedios son los sistemas de creencias y formas de expresión verbal y actitudinal que inciden en la forma como se procesa la información; para (Beck y Clark, 1988) son "estructuras funcionales de representaciones relativamente duraderas del conocimiento y la experiencia anterior” (pp. 382).

Los nucleares, funcionan a un nivel más profundo de la interacción neuronal, por lo que inciden en mayor medida en el procesamiento de la información. Los esquemas intermedios, inciden sobre el significado de experiencias y condicionan la manera como se interpreta, comprende y se actúa en la realidad (Sanz y Vázquez, 2008). Mientras que, los pensamientos voluntarios/automáticos son ideas de orden jerárquico que operan en la memoria humana y se producen de manera automática, natural y espontánea.

Cuando existen factores externos que afectan uno de estos componentes, se ve afectada la memoria. El asunto se vuelve peligroso, cuando la memoria deja de recibir información e incorpora información falsa, distorsionada o reduce su capacidad de asimilación. Se deben desarrollar diferentes mecanismos para producir la rehabilitación cognitiva, entre ellos: Restauración, Compensación, Sustitución, Activación-estimulación e Integración. Se pueden desarrollar a través de diferen- 
tes modalidades: Estimulación no dirigida, Entrenamiento de procesos específicos, Mejora de la salud física, emocional y del funcionamiento social. Así mismo, se pueden usar las técnicas del priming visual y semántico, para que sirvan de elemento activador en la conciencia del estudiante, al dimensionar aspectos negativos de los efectos del problema de aprendizaje que está apareciendo.

Para medir su evolución, se realiza una evaluación neurocognitiva que permita establecer los momentos de cambio durante la recuperación, siendo en todo momento necesario la utilización de pruebas cuantitativas y cualitativas estandarizadas, para comparar la evolución del proceso. Se deben controlar con precisión, variables como el tiempo de exposición de los estímulos y el tiempo de reacción, la respuesta y la evolución de la respuesta, así como el aumento o no de palabras hostiles o violentas y de palabras relacionadas con factores positivos o valores como la esperanza, la paz y los derechos humanos.

El olvido en la educación, tiene un valor importante y la carencia de un claro mapa cognitivo y conceptual incide para que se complete la comprensión en el tejido de pensamiento. Todo vacío o imprecisión es un error en la programación del sujeto para codificar la realidad, entenderla y actuar en ella, en especial, en campos donde la demanda de conocimientos integrados lo exige. Así que, validar el priming visual y semántico, es una forma de viabilizar en la comprensión del estudiante, un mecanismo de pensamiento que le permita generar formas instantáneas de respuesta desde la operación de sus campos semánticos mentales más sanos y estables y no desde las zonas neutras o negativas del cerebro.

En este sentido, el uso del priming visual o semántico, es una estrategia de generación o planificación de respuesta que sirve como elemento de anclaje para recuperar información y organizarla y funciona mejor, cuando las respuestas o conductas generadas son apropiadas o se adaptan a cada tarea. Por otro lado, Anderson y Bower (1974) sostienen que la decodificación produce una múltiple representación de la información dependiendo del sujeto y, de esta manera, se discriminan los significados que adquiere del contexto y de la representación de la palabra sobre el significado de esa palabra y su relación en un contexto semántico-cognitivo más amplio; esto con el fin de establecer los efectos entre los ítems a un nivel macroestructural y supraestructural.

Por ser un tema complejo y multicausal, se debe realizar un acompañamiento a las instituciones que tienen contextos y clima interno de violencia escolar, aplicar los diagnósticos para establecer el nivel de desarrollo cognitivo e identificar los posibles estudiantes, con dificultades en memoria sobre cultura de la legalidad, ya que son población de riesgo y, en especial, hacia ellos deben dirigirse acciones pedagógicas y preventivas.

En este campo, se deben abordar acciones con pedagógica específica, a partir de las estrategias de transformación. Según Román y Gallego (1994) hay cinco 
grupos de estrategias de aprendizaje: a) Estrategias de adquisición de información, b) Estrategias de codificación de información, c) Estrategias de recuperación de información, d) Estrategias socioafectivas, y e) Estrategias metacognitivas. Alternar estas estrategias, implica favorecer la búsqueda de información en la memoria y la generación de respuesta, implica promover el desarrollo de habilidades y saberes para controlar la ansiedad, las expectativas, los distractores y para promover la solidaridad y la cooperación.

\section{Conclusiones}

El uso de inducción semántica a través del reconocimiento y priming visual en el aprendizaje de la cultura de la legalidad, es un método que incide en la estructuración cognitiva del estudiante y para su efectividad depende del grado de superposición perceptiva entre el primer estímulo y los estímulos subsiguientes. Se establece que el priming refleja una forma general de aprendizaje en los sistemas de representación perceptiva (Tulving y Schacter, 1990), que puede producir modificaciones cognitivas profundas; su empleo en la educación sirve para la incorporación de nuevas expresiones en el vocabulario cotidiano, en lo cual la forma del priming es determinante para la accesibilidad a los conceptos que sólo pueden ser manejados como resultado del aprendizaje en sistemas de representación semántica. Y para ello, queda demostrado el priming conceptual con narrativa trágica, que conduce a una facilitación del procesamiento del significado de una palabra.

Se concluye también que el formato de presentación de los estímulos es mucho más llamativo cuando se emplean elementos en movimiento, con fondo musical, siendo un estimulante que aumenta la motivación.

Finalmente, su empleo en el aula permite ofrecer un feedback rápido y correcto, para incidir en el sistema interactivo de cambios de ideas del estudiante en función de las respuestas y su corrección y del arraigo que pueda presentar al pensamiento distorsionado. De tal manera que permite controlar con precisión y modificar, si es necesario, ciertos parámetros de las tareas tales como el número de estímulos, el tiempo de exposición de éstos y el nivel de dificultad. 


\section{Referencias}

Anderson, J.; Bower, G. H. (1974). A Propositional Theory of Recognition Memory. Memory and Cognition, 2, 406-412. https://link.springer.com/ content/pdf/10.3758/BFo3196896.pdf [Consultado el 23 de mayo de 2018 ].

Baddeley, A. D. (1999). Psicología cognitiva: un curso modular. Fundamentos de la memoria humana. Hove, Inglaterra: Psychology Press/Taylor \& Francis.

Baron, J. C.; Eustache, F. (2001). An ERD Mapping Study of the Neurocognitive Processes Involved in the Perceptual and Semantic Analysis of Environmental Sounds and Words. Cognitive Brain Research, 11(2), 235-248. https://www.ncbi.nlm.nih.gov/pubmed/11275485 [Consultado el 6 de mayo de 2018].

Beck, A.; Clark, D. (1988). Anxiety and Depression: An Information Processing Perspective. Anxiety Research, 1(1), 23-36. https://www.tandfonline. com/doi/abs/10.1080/10615808808248218 [Consultado el 8 de mayo de 2018 ].

Beck, J. S. (2005). Cognitive Therapy for Challenging Problems: What to do When the Basics don't Work. New York: The Guilford Press.

Bloomquist, M. L.; Schnell, S. V. (2005). Helping Children with Aggression and Conduct Problems. Best Practices fo Intervention. New York, EEUU: Guilford Press.

Broadbent, E. (1958). Perception and Communication. London, England: Pergamon Press.

Bruner, J. (1988). Realidad mental y mundos posibles. Los actos de la imaginación que dan sentido a la experiencia. Barcelona, España: Gedisa.

Calvete, E. (2008). Justification of Violence and Grandiosity Schemas as Predictors of Antisocial Behavior in Adolescents. Journal of Abnormal Child Psychology, 36 (2008), 1083-1095. https://link.springer.com/article/10.1007\%2Fs 10802-008-9229-5 
Calvete, E.; Orue, I.; Estévez, A.; Villardón, L.; Padilla, P. (2014). La rumia melancólica como mediador en la relación entre los primeros esquemas desadaptativos y los síntomas de depresión y ansiedad social en adolescentes. Barcelona, España: Masson, Ed.

Campbell, R.; Conway, M. (1995). Broken Memories. Londres, Inglaterra: Blackwell.

Déry, M.; Toupin, J.; Pauzé, R.; Mercier, H.; Fortin, L. (1999). Neuropsychological Characteristics of Adolescents with Conduct Disorder: Association with Attention-deficit-hyperactivity and Aggression. J Abnorm Child Psycho 27(3), 225-36. https://link.springer.com/article/10.1023\%2FA\%3A1021904523912 [Consultado el 4 de mayo de 2018].

Diamond, R.; Carey, S. (1977). Developmental Changes in the Representation of Faces. Journal of Experimental Child Psychology, 23, 1-22. https://www. sciencedirect.com/science/article/abs/pii/o022096577900698 [Consultado el 4 de mayo de 2018].

Elias, N. (1990). Compromiso y distanciamiento. Barcelona, España: Península.

Felser, C.; Roberts, L. (2007). Processing Wh-dependencies in a Second Language: A Crossmodal Priming Study. Second Language Research, 23, 9-36. https://journals.sagepub.com/doi/10.1177/0267658307071600 [Consultado el 4 de mayo de 2019$]$.

Froufe, M. (1997). Inconsciente cognitivo: La cara oculta de la mente. Madrid, España: Biblioteca Nueva.

García-Bajos, E.; Migueles, M.; Anderson, M. C. (2009). El conocimiento del guion modula el olvido inducido por la recuperación para eventos de testigos oculares (Tesis de grado, inédita). Facultad de Psicología, Universidad del País Vasco. San Sebastián, España.

Geddes, H. (2010). El apego en el aula. Relaciones entre las primeras experiencias infantiles, el bienestar emocional y el rendimiento escolar. Barcelona, España: Grao. 
Gómez-Ariza, C. J.; Lechuga, M. T.; Pelegrina, S.; Bajo, M. T. (2005). Retrieval-induced Forgetting in Recall and Recognition of Thematically Related and Unrelated Sentences. Memory E Cognition, 33, 1431-1441. https:// link.springer.com/content/pdf/ 10.3758\%2FBF03 193376.pdf [Consultado el 4 de mayo de 2019].

Greene, M. R.; Oliva, A. (2009). High-level Aftereffects to Natural Scenes. Journal of Vision, 8(6), 1104. https://jov.arvojournals.org/article.aspx?articleid $=2136700$ [Consultado el 4 de mayo de 2019].

Huesmann, L. R.; Guerra, N. G.; Miller, L.; Zelli, A. (1992). The Role of Social Norms in the Development of Aggression. In H. Zumkley \& A. Fraczek (Eds.). Socialization and Aggression (pp. 139-151). New York: Springer-Verlag.

Inheler, B.; Sinclair, H.; Bovet, M. (1975). Learning and Developmen of Cognition. Cambridge, Massachusetts: Harvard University Press.

Luna, K.; Migueles, M. (2008). Typicality and Misinformation. Two Sources of Distortion. Psicológica, 29, 171-188. https://www.uv.es/psicologica/articulos2.08/4LUNA.pdf [Consultado el 6 de mayo de 2018 ].

Macbeth, G.; López-Alonso, A. O. (2007). Aportes del enfoque ecológico a los estudios sobre calibración. Acta Psiquiátrica y Psicológica de América Latina, 53(1), 55-61. http://www.acta.org.ar/O4-WebForms/frmVolumenes. aspx? DatVolumen $=7 \&$ Palabra $=\& I d A$ bonado $=[$ Consultado el 6 de mayo de 2018].

Manzanero, A. L. (2008). Psicología del testimonio. Una aplicación de los estudios sobre la memoria. Madrid, España: Pirámide.

Mulligan, N. W.; Hornstein, S. L. (2000). Atención y cebado perceptivo en la tarea de identificación perceptiva. Journal of Experimental Psychology: Learning, Memory, and Cognition, 26(3), 626-637. https://psycnet.apa.org/ record/2000-03416-005 [Consultado el 6 de mayo de 2019].

Nadel, L.; Campbell, J. L.; Ryan, L. (2007). Autobiographical Memory Retrieval and Hippocampal Activation as a Function of Repetition and the Passage of Time. Neural Plasticity, 1-14. http://downloads.hindawi.com/journals/ np/2007/090472.pdf [Consultado el 6 de mayo de 2018]. 
Nilsson, L. G.; Law, J.; Tulving, E. (1988). Recognition Failure of Recallable Unique Names: Evidence for an Empirical Law of Memory and Learning. Journal of Experimental Psychology: Learning, Memory E Cognition, 14(2), 266-277. https://psycnet.apa.org/doiLanding?doi=10.1037\% 2Fo278-7393.14.2.266 [Consultado el 4 de mayo de 2018].

Rhodes, G.; Brake, S.; Carey, S. (1987). Identification and Ratings of Caricatures: Implications for Mental Representation of Faces. Cognitive Psychology, 19, 473-497. https://www.sciencedirect.com/science/article/ pii/0010028587900168 [Consultado el 4 de mayo de 2018].

Román, J. M.; Gallego, S. (1994). ACRA: Escala de estrategias de aprendizaje. Madrid, España: TEA Ediciones.

Ruchkin, V.; Schwab M; Koposov R; Vermeiren R; Steiner H. (2002). Violence Exposure Postraumatic Stree, and Personality in Juvenile Delinque. Adolece Psychiatry, 41, 322-329. https://www.ncbi.nlm.nih.gov/pub$\mathrm{med} / 11886027$ [Consultado el 4 de mayo de 2018].

Sanz, J.; Vázquez, C. (2008). Trastornos del estado de ánimo: teorías psicológicas. En A. Belloch, B. Sandín y F. Ramos (Eds.). Manual de Psicopatología (pp. 271-298). Madrid, España: McGraw-Hill.

Schacter, D. L.; Tulving, E. (1994). ¿Cuáles son los sistemas de memoria de 1994? En D. L. Schacter y E. Tulving (Eds.). Memory systems (pp. 1-38). Cambridge, EE UU: The MIT Press.

Schott, B.; Richardson-Klavehn, A.; Heinze, H. J.; Düzel, E. (2002). Perceptual priming versus explicit memory: Dissociable Neural Correlates at Encoding. Journal of Cognitive Neuroscience, 14, 578-592. https://www.ncbi. nlm.nih.gov/pubmed/12126499 [Consultado el 4 de mayo de 2018].

Schott, B.; Henson, R. N.; Richardson-Klavehn, A.; Becker C.; Thoma, V.; Heinze, H. J.; Düzel, E. (2004). Redefining Implicit and Explicit Memory: The Functional Neuroanatomy of Priming, Remembering, and Control of Retrieval. Proceedings of the National Academy of Sciences, 102, 1257-1262. https://www.pnas.org/content/102/4/1257 [Consultado el 4 de mayo de 2018 ]. 
Schott, B.; Richarson-Klavehn, A.; Henson, N. A.; Becker, C.; Heinze, H. J.; Düzel, E. (2006). Neuroanatomical dissociation of encoding processes related to priming and explicit memory. The Journal of Neuroscience, 26, 792-800. https://www.jneurosci.org/content/26/3/792 [Consultado el 4 de mayo de 2018].

Siegler, R. S. (1996). Emerging Mind. The Process of Change in Childres Thinking. New York, EEUU: Osford University Press.

Slaby, R. G.; Guerra, N. G. (1988). Mediadores cognitivos de la agresión en delincuentes adolescentes. Psicología del desarrollo, 24(4), 580-588. https:// psycnet.apa.org/record/1989-01839-001 [Consultado el 4t de mayo de 2018].

Strauss, A.; Corbin, J. (1998). Basics of Qualitative Research: Techniques and Procedures for Developing Grounded Theory. Los Ángeles, California: Thousand Oaks.

Tulving, E.; Schacter, D. L. (1990). Priming and Human Memory Systems. Science, 247(4940), 301-306. https://www.ncbi.nlm.nih.gov/pubmed/2296719 [Consultado el 23 de mayo de 2018].

Turecki, S.; Tonner, L. (2003). El niño difícil. Barcelona, España: Ediciones Medici.

Wald, J.; M. Kurlander (2003). Connected in Seattle? An Exploratory Study of Student Perceptions of Discipline and Attachments to Teachers. New York, EE UU: New Directions for Youth Development. 
